\title{
A INFLUÊNCIA DO RUÍDO AMBIENTAL NO DESEMPENHO DE ESCOLARES NOS TESTES DE PADRÃO TONAL DE FREQUÊNCIA E PADRÃO TONAL DE DURAÇÃO
}

\author{
Environmental noise influence on student performance \\ in the Frequency Pattern Tests and Duration Pattern Tests
}

Ludimila Souza Nascimento ${ }^{(1)}$, Stela Maris Aguiar Lemos ${ }^{(2)}$

\begin{abstract}
RESUMO
Objetivo: caracterizar o desempenho de escolares nos testes de padrão tonal de frequência e padrão tonal de duração no silêncio e na presença de ruído ambiental. Metodo: trata-se de estudo experimental transversal, com amostra de conveniência, composta por 70 estudantes, que responderam a um formulário de percepção do ruído ambiental. O nível de ruído da escola foi avaliado com medidor de nível de pressão sonora. Os estudantes foram submetidos à avaliações (fala, motricidade orofacial, linguagem e simplificada do processamento auditivo) e distribuídos em dois grupos (G1 sem alteração fonoaudiológica e G2 com alteração fonoaudiológica). Foram realizados também os testes de padrão tonal de frequência e duração (silêncio e ruído). Resultados: o nível médio de pressão sonora da escola variou de 57,2 $\mathrm{dB}(\mathrm{A})$ na sala de informática a 83,6 $\mathrm{dB}(\mathrm{A})$ na quadra de esportes. Segundo os estudantes, o que mais interfere em suas atividades é o barulho de conversas durante a aula. Quanto aos testes de padrão tonal de frequência (TPF) e duração (TPD), observou-se que a média de acertos no ambiente silencioso foi maior que no ambiente ruidoso. O G1 apresentou melhor desempenho no TPD e TPF que G2. No ruído houve piora no desempenho dos dois grupos (G1 e G2) nos testes. Conclusão: os níveis de pressão sonora da escola encontram-se elevados e fora do padrão recomendado pelas normas nacionais. No ruído, houve piora no desempenho dos testes nos dois grupos estudados.
\end{abstract}

DESCRITORES: Ruído; Testes Auditivos; Aprendizagem; Efeitos do Ruído; Percepção Auditiva

\section{INTRODUÇÃO}

A poluição sonora é um problema ambiental que tem se destacado pela grande influência na saúde física e emocional do indivíduo ${ }^{\circledR}$. O ruído é um poluente invisível que prejudica a qualidade de vida da população ${ }^{2-4}$ e tem um papel importante para

(1) Fonoaudióloga pela Universidade Federal de Minas Gerais; Mestranda do Programa de Pós-Graduação em Estudos Linguísticos da Universidade Federal de Minas Gerais UFMG, Belo Horizonte, Brasil.

(2) Fonoaudióloga; Professora adjunto do Departamento de Fonoaudiologia da Universidade Federal de Minas Gerais - UFMG, Belo Horizonte, Brasil; Doutora em ciencias pelo Programa de Pós-Graduação em Distúrbios da Comunicação Humana do Departamento de Fonoaudiologia da Universidade Federal de São Paulo.

Conflito de interesses: inexistente o desenvolvimento de problemas auditivos ${ }^{5-8}$. No ambiente escolar, em específico, pode ocasionar danos ao processo de ensino-aprendizagem, por interferir na realização de atividades ${ }^{9,10}$.

Os estudantes e os professores são prejudicados por elevados níveis sonoros em sala de aula. Desse modo, atividades escolares que exigem uma maior concentração são difíceis de serem realizadas em ambiente ruidoso.

Para que a aprendizagem seja efetiva, o ambiente deve ser propício ao desenvolvimento global do indivíduo, com boa acústica, iluminação, ventilação. Características que nem sempre estão presentes no meio acadêmico ${ }^{11}$.

A Fonoaudiologia, enquanto ciência, investiga os danos ocasionados pelo ruído à saúde auditiva da população e também os seus efeitos na comunicação humana. Um processo comunicativo 
eficiente exige que a mensagem seja compreendida, e quando existe falha nessa recepção, podem ocorrer alterações de linguagem e do processamento da informação sonora.

O processamento auditivo corresponde aos mecanismos do sistema auditivo envolvidos nos fenômenos comportamentais de localização e lateralização da fonte sonora; discriminação auditiva; reconhecimento de padrões auditivos; aspectos temporais da audição; desempenho auditivo na presença de sinais acústicos em competição; e desempenho auditivo com sinais acústicos degradados. Entre as habilidades relacionadas aos aspectos temporais da audição ou processamento auditivo temporal, são incluídos o mascaramento, a integração, a ordenação e a resolução temporais ${ }^{12}$. A percepção dos estímulos sonoros de maneira adequada é importante para um bom desempenho escolar. A avaliação das habilidades do processamento auditivo tem contribuído para a identificação e intervenção em crianças com dificuldades escolares.

Crianças e adolescentes são mais vulneráveis à interferências em seu processo de aprendizagem, visto que se encontram em processo de desenvolvimento das habilidades auditivas e também no período de aquisição e desenvolvimento da linguagem oral e escrita ${ }^{13}$.

Os testes comportamentais para avaliar o processamento auditivo denominados de padrão tonal de frequência e padrão tonal de duração avaliam habilidades importantes para o desenvolvimento da leitura e escrita e, consequentemente, para um bom desempenho escolar.

Estudar a influência do ruído ambiental no processo de ensino-aprendizagem é importante para fundamentar a prática fonoaudiológica em escolas. É necessário investigar os fatores que podem interferir no aprendizado de escolares. Baseado nesses conhecimentos, pode-se realizar intervenções mais efetivas e propor programas de conservação auditiva em escolas.

$\mathrm{Na}$ prática clínica fonoaudiológica, frequentemente são atendidos escolares com queixas de aprendizagem, que podem ser agravadas ou ocasionadas pela exposição diária a ruídos em sala de aula.

Diante do exposto e em decorrência da escassez de investigações que correlacionem a influência do ruído ambiental no processamento da informação auditiva no ambiente escolar, este trabalho tornase relevante para embasar a prática clínica fonoaudiológica e pesquisas futuras sobre o tema. Essa pesquisa poderá contribuir também para a reflexão da prática escolar, bem como a adoção de medidas de promoção da saúde em escolas.
Assim, o presente estudo tem por objetivos: caracterizar e comparar o desempenho de estudantes de ensino fundamental com e sem alterações fonoaudiológicas nos testes de padrão tonal de frequência e de padrão tonal de duração em escuta diótica, no ambiente escolar, em locais com diferentes níveis de ruído segundo as variáveis sexo e presença de alterações fonoaudiológicas; e verificar a associação entre o desempenho nestes testes e a percepção dos estudantes quanto ao ruído no ambiente escolar.

\section{MÉTODO}

Este estudo, do tipo experimental transversal, com amostra de conveniência, foi realizado em uma escola de ensino fundamental da rede pública de ensino de Belo Horizonte. Pais e adolescentes assinaram o Termo de Consentimento Livre e Esclarecido, para a participação voluntária no estudo conforme a resolução 196/96 do Conselho Nacional de Saúde.

Para o cálculo do tamanho da amostra foi considerado o número total de sujeitos na escola segundo a faixa etária e sexo. Assim, foi considerada amostra aleatória estratificada segundo sexo e faixa etária.

Os critérios de inclusão para a seleção dos estudantes foram: ter idade entre 10 e 15 anos; não apresentar evidências ou histórico de alterações cognitivas, neurológicas ou motoras; retornar com o termo de consentimento livre e esclarecido assinado pelos próprios estudantes e por seus responsáveis. Foram considerados critérios de exclusão: Não conseguir realizar os testes propostos; desistir durante a aplicação das avaliações; estar em processo de avaliação ou terapia fonoaudiológica; apresentar diagnóstico prévio de alteração auditiva; não responder ou responder apenas parcialmente ao formulário de percepção do ruído ambiental.

Foram excluídos da amostra sete estudantes: um estudante desistiu durante a aplicação das avaliações e o outro apresentou evidências e histórico de alteração cognitiva, neurológica e motora. Os outros cinco estudantes não concluíram as avaliações devido a faltas durante o período letivo.

Atenderam, portanto, aos critérios de inclusão 70 estudantes. Assim, a amostra desse estudo constituiu-se de 70 estudantes, sendo $40 \%(n=28)$ do sexo masculino e $60 \%(n=42)$ do sexo feminino, na faixa etária de 10 a 15 anos.

Inicialmente, os estudantes responderam ao formulário de percepção do ruído ambiental, elaborado pelas pesquisadoras, na própria sala de aula, composto por oito questões, com os seguintes eixos temáticos: mapeamento dos locais ruidosos e escala visual analógica de quantificação da 
interferência do ruído, assim como a sua percepção e causas.

No formulário de percepção do ruído ambiental, foram fornecidas opções com as causas mais frequentes de ruído elevado em sala de aula e também uma escala visual analógica para quantificação da interferência de cada uma dessas causas na atenção em sala de aula (escala de $0-10$ ).

Após a aplicação do formulário, foi iniciada a avaliação dos seguintes aspectos: fala, linguagem, motricidade orofacial e avaliação simplificada do processamento auditivo. Todas essas avaliações foram realizadas, individualmente, em sala cedida pela escola.

A avaliação simplificada de linguagem foi realizada por meio da aplicação de sequência lógicotemporal, para que o indivíduo descrevesse oralmente e também escrevesse o conteúdo presente na sequência de gravuras.

Para a avaliação de fala, utilizou-se o álbum fonêmico, no qual foram registrados todos os fonemas presentes, assim como substituições, omissões ou distorções realizadas durante a emissão de palavras foneticamente balanceadas.

No intuito de garantir que todos os aspectos fonoaudiológicos fossem triados optou-se por realizar, também a avaliação da motricidade orofacial, incluindo a observação do aspecto de palato, dentes e classificação da oclusão; tensão e mobilidade de lábios e língua por meio de contra-resistência com espátula e movimentos de retração, protrusão e lateralização de língua, protrusão e retração de lábios; estalo e vibração dessas estruturas.

$\mathrm{Na}$ Avaliação simplificada do processamento auditivo, realizou-se os testes de memória sequencial verbal e não-verbal e localização sonora. Além disso, foi realizada pesquisa do reflexo cócleopalpebral (RCP) a $100 \mathrm{~dB}$ NPS para excluir perdas auditivas bilaterais de graus severo e profundo ${ }^{14}$.

Para a avaliação dos níveis de pressão sonora da escola, foi utilizado o medidor de nível de pressão sonora da marca Homis, modelo 232, calibrado em seis de fevereiro de 2009 , com certificado de calibração no 807.31/09. As medições foram realizadas de acordo com os procedimentos da norma brasileira NBR $10.151^{15}$. O nível de ruído foi avaliado na quadra de esportes durante a aula de educação física; no pátio da escola durante o recreio e no período habitual das aulas; na biblioteca; na sala de informática; no auditório da escola; em sala de aula, durante a aula, com a presença dos estudantes, e no recesso escolar, na ausência dos estudantes. As medições foram realizadas pela manhã com intervalos de uma hora, durante um período de quatro horas, em todos os locais e situações pré-determinados.
A aplicação dos testes de padrão tonal de duração e padrão tonal de frequência foi realizada em escuta diótica e em grupos de quatro indivíduos conforme proposta da literatura ${ }^{17}$. Vale ressaltar que a utilização dos testes em campo livre se justifica por avaliar a audição em diferentes meios ambientes para propósitos de comunicação - "audição social" /audição natural ${ }^{16}$.

Cada grupo foi submetido aos testes em duas situações: em um local silencioso e em outro com presença de ruído ambiental. $\mathrm{O}$ auditório da escola foi escolhido como ambiente ruidoso por apresentar $70,1 \mathrm{~dB}(\mathrm{~A})$ e a sala de informática foi selecionada como local silencioso para as testagens, por apresentar 57,2 dB(A) de nível de pressão sonora. $O$ local com menor nível de ruído foi chamado de silencioso para facilitar a discussão dos dados.

No teste de padrão tonal de frequência (TPF) foram apresentadas sequências com três (TPF3) e quatro (TPF4) estímulos, com combinações de frequência baixa $(440 \mathrm{~Hz})$ e alta $(493 \mathrm{~Hz})$ e duração fixa. O teste de padrão tonal de duração (TPD) seguiu procedimento semelhante ao TPF, porém com diferenças quanto à duração, sendo constituído de tons de duração longa (161ms) e curta (59ms) e frequência fixa $(440 \mathrm{~Hz})$. No total foram apresentados 40 itens, subdivididos em 20 itens para avaliação do padrão tonal de duração e 20 itens para o padrão tonal de frequência. Em cada subdivisão havia 10 sequências com três estímulos e 10 com quatro estímulos combinados de diferentes formas. Optou-se pela realização dos testes de padrão tonal de frequência e padrão tonal de duração em campo livre e em grupos de quatro indivíduos, por ser uma avaliação de fácil aplicação, baixo custo e possível de ser realizada no ambiente escolar. Cabe ressaltar que a metodologia e a efetividade da aplicação utilizada no presente estudo foram descritas na literatura nacional ${ }^{17}$.

Cada teste foi realizado duas vezes em cada situação avaliada. Na primeira vez, os estudantes realizaram a marcação de suas respostas com símbolos. Para a marcação, eles representaram os estímulos longo e curto, respectivamente, com um traço e um ponto. Já os estímulos alto e baixo foram representados pelos símbolos $\mathrm{X}$ e $\mathrm{O}$, respectivamente. Na segunda vez foi realizada a resposta por nomeação, os estudantes escreveram em uma folha o nome do estímulo (curto/longo, alto/baixo). Estas modalidades de resposta foram selecionadas devido a aplicação dos testes ser realizada em grupo. A realização dos testes foi de forma randomizada, em parte dos estudantes iniciou-se os estímulos em situação de silêncio e em parte da amostra em situação de ruído.

Para emissão dos estímulos foi utilizado o programa Windows Media Player em um 
computador da marca Intelbrás, com volume fixo e caixas de som posicionadas a um metro de distância dos ouvintes.

Os estudantes foram distribuídos em dois grupos: grupo sem alteração fonoaudiológica (G1) e grupo com alteração fonoaudiológica (G2). Os estudantes do G2 falharam em pelo menos uma das avaliações realizadas (fala, linguagem, sistema sensório-motor oral e avaliação simplificada do processamento auditivo) e os do G1 obtiveram respostas adequadas em todos os procedimentos realizados. O G1 foi composto por 41 estudantes e o G2 por 29 (Tabela 1). O desempenho do G1 e G2 foi comparado quanto a realização dos testes de padrão tonal de frequência e padrão tonal de duração.

Este estudo teve a aprovação do Comitê de Ética em pesquisa da UFMG, sob parecer nํㅡㄴ ETC 640/08.

Para realizar a comparação dos testes de padrão tonal de frequência e padrão tonal duração entre os ambientes, silencioso e ruidoso, foi aplicado o teste Não Paramétrico de Wilcoxon. O teste de Mann-Whitey foi utilizado para comparar os testes de processamento auditivo, tanto no ambiente silencioso quanto no ambiente ruidoso, e a variável alterações fonoaudiológicas. $\mathrm{Na}$ associação entre o formulário de percepção do ruído e os testes de padrão tonal de frequência e duração foram utilizados modelos lineares com distribuição de erro de Poisson, o testes Qui-Quadrado por simulação de Monte Carlo, Teste de correlação de Spearman e Teste de Mann-Whitney. Considerou-se, para o estudo, o nível de significância menor do que 0,05.

\section{RESULTADOS}

Considerando o questionário de percepção do ruído ambiental, a maior média das marcações foi obtida, em ambos os grupos, para pessoas conversando, sendo em G1 de 7,92 e em G2 de 7,48 , e a menor média para buzinas. O teste de
Mann-Whitney mostrou a existência de associação com significância estatística entre G1 e G2 apenas para a variável buzinas, o que não foi verificado para as outras causas investigadas, a saber: pessoas conversando, arrastar de mesas e cadeiras, porta batendo e sirene da escola (Tabela 2).

$\mathrm{Na}$ avaliação do ruído ambiental da escola, constatou-se que a quadra de esportes foi o local mais ruidoso, com nível de pressão sonora médio de $83,6 \mathrm{~dB}(\mathrm{~A})$, e o local menos ruidoso foi a sala de informática, com 57,2 dB (A). No interior das salas de aula avaliadas foram encontrados níveis sonoros de 68,1 dB(A) (Figura 1).

No teste de padrão tonal de frequência foi verificada associação com significância estatística entre o TPF3 (símbolos) e o local da testagem. Já para o TPF3 (nomeação) no ambiente silencioso e ruidoso não foi observada relação com significância estatística. No TPF4 não foi verificada associação com significância estatística para os dois tipos de resposta e local de realização dos testes. Vale destacar que, conforme a literatura nacional ${ }^{17}$, foram utilizados como critérios de referência de adequação: para o TPF3 sete acertos (70\% de acertos) e para o TPF4 seis acertos, $60 \%$ de acertos; para o TPD3 dez acertos (100\%) e TPD4 nove acertos (90\%).

Para o teste de padrão tonal de duração foi encontrada relação com significância estatística para o TPD3 e TPD4, nas duas modalidades de resposta, para o ambiente silencioso e ruidoso.

$\mathrm{Na}$ análise do TPF e TPD e os grupos G1 e G2, observou-se que o desempenho médio nesses testes, tanto na modalidade de resposta com símbolos quanto nomeação, foram maiores no grupo sem alteração fonoaudiológica (G1). Esse mesmo padrão foi observado nos ambientes silencioso e ruidoso. Houve associação com significância estatística para o TPF3, TPD3 e TPD4, todos no ambiente silencioso e para a modalidade de resposta nomeação (Tabela 5).

Tabela 1 - Distribuição da amostra segundo grupo e sexo

\begin{tabular}{lcccccc}
\hline & \multicolumn{9}{c}{ Sexo } \\
& \multicolumn{2}{c}{ Feminino } & \multicolumn{2}{c}{ Masculino } & \multicolumn{2}{c}{ Total } \\
\cline { 2 - 7 } & $\mathbf{N}$ & $\%$ & $\mathbf{N}$ & $\%$ & $\mathbf{N}$ & $\%$ \\
\hline G1 & 24,00 & 34,30 & 17,00 & 24,30 & 41,00 & 58,60 \\
G2 & 18,00 & 25,70 & 11,00 & 15,70 & 29,00 & 41,40 \\
\hline Total & 42,00 & 60,00 & 28,00 & 40,00 & 70,00 & 100,00 \\
\hline Legenda: & \multicolumn{7}{c}{} & & \\
N= Número & & & & & \\
G1: Grupo sem alteração fonoaudiológica & & & &
\end{tabular}


Tabela 2 - Medidas estatísticas da percepção dos níveis de ruído e associação entre escala visual analógica para quantificação da interferência do ruído na atenção em sala de aula - por grupo

\begin{tabular}{lccccccc}
\hline & Grupo & Mínimo & Máximo & Mediana & Média & DP & p-valor \\
\hline \multirow{2}{*}{ Pessoas Conversando } & G1 & 1,00 & 10,00 & 8,00 & 7,92 & 2,20 & \multirow{2}{*}{0,46} \\
& G2 & 2,00 & 10,00 & 9,00 & 7,48 & 2,42 & \\
\hline \multirow{2}{*}{ Cadeiras e mesas } & G1 & 1,00 & 10,00 & 6,00 & 6,19 & 2,73 & \multirow{2}{*}{0,70} \\
\hline \multirow{2}{*}{ Portastando batendo } & G2 & 1,00 & 10,00 & 7,00 & 6,44 & 2,83 & \\
& G1 & 0,00 & 10,00 & 3,00 & 3,75 & 2,69 & \multirow{2}{*}{0,14} \\
\multirow{2}{*}{ Buzinas } & G2 & 1,00 & 10,00 & 5,00 & 4,79 & 2,92 & \\
\hline \multirow{2}{*}{ Sirene da escola } & G1 & 0,00 & 10,00 & 1,00 & 2,34 & 3,15 & \multirow{2}{*}{$\mathbf{0 , 0 2 *}$} \\
& G2 & 0,00 & 10,00 & 3,00 & 3,62 & 3,06 & \multirow{2}{*}{0,07} \\
\hline
\end{tabular}

Teste de Mann Whitney

Legenda:

G1: Grupo sem alteração fonoaudiológica

G2: Grupo com alteração fonoaudiológica

DP: Desvio-padrão

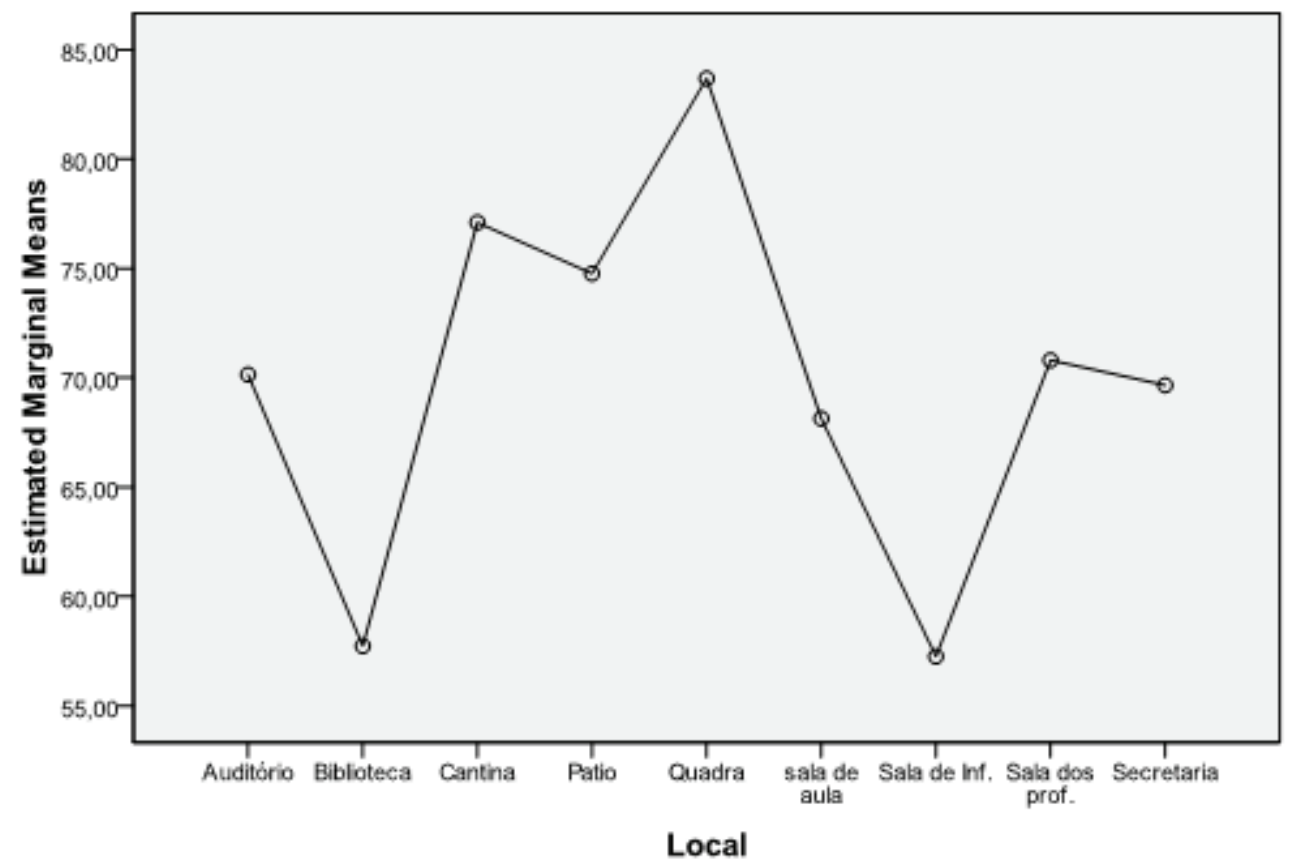

Figura 1 - Gráfico representativo dos valores médios de ruído ambiental de acordo com o local da escola 
Tabela 3 - Medidas estatísticas descritivas do desempenho da amostra total no teste de padrão tonal de frequência (em número de acertos)

\begin{tabular}{|c|c|c|c|c|c|c|}
\hline Variáveis & Mínimo & Máximo & Mediana & Média & DP & p-valor \\
\hline $\begin{array}{l}\text { TPF3 } \\
\text { Símbolos } \\
\text { Ambiente Silencioso }\end{array}$ & 1,00 & 10,00 & 4,00 & 4,41 & 2,44 & \multirow{2}{*}{$0,02^{*}$} \\
\hline $\begin{array}{l}\text { TPF3 } \\
\text { Símbolos } \\
\text { Ambiente Ruidoso }\end{array}$ & 0,00 & 10,00 & 3,00 & 3,74 & 2,22 & \\
\hline $\begin{array}{l}\text { TPF3 } \\
\text { Nomeação } \\
\text { Ambiente Silencioso }\end{array}$ & 1,00 & 10,00 & 3,00 & 4,03 & 2,81 & \multirow{2}{*}{0,28} \\
\hline $\begin{array}{l}\text { TPF3 } \\
\text { Nomeação } \\
\text { Ambiente Ruidoso } \\
\end{array}$ & 0,00 & 10,00 & 3,00 & 3,67 & 2,80 & \\
\hline $\begin{array}{l}\text { TPF4 } \\
\text { Símbolos } \\
\text { Ambiente Silencioso }\end{array}$ & 1,00 & 10,00 & 3,00 & 4,03 & 2,95 & \multirow{2}{*}{0,11} \\
\hline $\begin{array}{l}\text { TPF4 } \\
\text { Símbolos } \\
\text { Ambiente Ruidoso }\end{array}$ & 0,00 & 10,00 & 3,00 & 3,70 & 3,00 & \\
\hline $\begin{array}{l}\text { TPF4 } \\
\text { Nomeação } \\
\text { Ambiente Silencioso }\end{array}$ & 1,00 & 10,00 & 3,00 & 3,73 & 2,78 & \multirow{2}{*}{0,92} \\
\hline $\begin{array}{l}\text { TPF4 } \\
\text { Nomeação } \\
\text { Ambiente Ruidoso } \\
\end{array}$ & 0,00 & 10,00 & 3,00 & 3,70 & 2,24 & \\
\hline
\end{tabular}

Teste de Wilcoxon

Legenda:

TPF3: teste de padrão tonal de frequência com três sons

TPF4: teste de padrão tonal de frequência com quatro sons

DP: Desvio padrão 
Tabela 4 - Medidas estatísticas descritivas do desempenho da amostra total no teste de padrão tonal de duração (em número de acertos)

\begin{tabular}{|c|c|c|c|c|c|c|}
\hline Variáveis & Mínimo & Máximo & Mediana & Média & DP & $\overline{p-v a l o r}$ \\
\hline $\begin{array}{l}\text { TPD3 } \\
\text { Símbolos } \\
\text { Ambiente Silencioso }\end{array}$ & 1,00 & 10,00 & 10,00 & 8,63 & 2,46 & \multirow{2}{*}{$0,00^{*}$} \\
\hline $\begin{array}{l}\text { TPD3 } \\
\text { Símbolos } \\
\text { Ambiente Ruidoso }\end{array}$ & 1,00 & 10,00 & 9,00 & 7,54 & 3,04 & \\
\hline $\begin{array}{l}\text { TPD3 } \\
\text { Nomeação } \\
\text { Ambiente Silencioso }\end{array}$ & 1,00 & 10,00 & 10,00 & 8,59 & 2,24 & \multirow{2}{*}{$0,01^{*}$} \\
\hline $\begin{array}{l}\text { TPD3 } \\
\text { Nomeação } \\
\text { Ambiente Ruidoso }\end{array}$ & 1,00 & 10,00 & 9,00 & 7,79 & 2,52 & \\
\hline $\begin{array}{l}\text { TPD4 } \\
\text { Símbolos } \\
\text { Ambiente Silencioso }\end{array}$ & 1,00 & 10,00 & 9,00 & 8,44 & 2,18 & \multirow{2}{*}{$0,03^{*}$} \\
\hline $\begin{array}{l}\text { TPD4 } \\
\text { Símbolos } \\
\text { Ambiente Ruidoso } \\
\end{array}$ & 0,00 & 10,00 & 9,00 & 7,81 & 2,79 & \\
\hline $\begin{array}{l}\text { TPD4 } \\
\text { Nomeação } \\
\text { Ambiente Silencioso }\end{array}$ & 2,00 & 10,00 & 9,00 & 8,31 & 2,22 & \multirow{2}{*}{$0,01^{*}$} \\
\hline $\begin{array}{l}\text { TPD4 } \\
\text { Nomeação } \\
\text { Ambiente Ruidoso }\end{array}$ & 0,00 & 10,00 & 9,00 & 7,63 & 2,74 & \\
\hline
\end{tabular}

Teste de Wilcoxon

Legenda:

TPD3: teste de padrão tonal de duração com três sons

TPD4: teste de padrão tonal de duração com quatro sons

DP: Desvio padrão

Tabela 5 - Medidas estatísticas do desempenho nos testes de padrão tonal de frequência e padrão tonal de duração em ambiente silencioso e ruidoso (nomeação) - segundo o grupo

\begin{tabular}{|c|c|c|c|c|c|c|c|c|c|c|c|c|c|}
\hline & \multirow{3}{*}{ Grupo } & \multicolumn{12}{|c|}{ Nomeação } \\
\hline & & \multicolumn{6}{|c|}{ Ambiente Silencioso } & \multicolumn{6}{|c|}{ Ambiente Ruidoso } \\
\hline & & Mínimo & Máximo & Mediana & Média & DP & p-valor & Mínimo & Máximo & Mediana & Média & DP & p-valor \\
\hline \multirow{2}{*}{ TPF3 } & G1 & 1,00 & 10,00 & 4,00 & 4,71 & 2,88 & \multirow{2}{*}{$0,01^{*}$} & 0,00 & 10,00 & 4,00 & 4,20 & 3,28 & \multirow{2}{*}{0,16} \\
\hline & G2 & 1,00 & 10,00 & 2,00 & 3,07 & 2,47 & & 0,00 & 8,00 & 3,00 & 2,93 & 1,77 & \\
\hline \multirow{2}{*}{ TPF4 } & G1 & 1,00 & 10,00 & 3,00 & 4,15 & 3,15 & \multirow{2}{*}{0,32} & 0,00 & 10,00 & 3,00 & 4,27 & 3,27 & \multirow{2}{*}{0,09} \\
\hline & G2 & 1,00 & 7,00 & 3,00 & 3,14 & 2,04 & & 0,00 & 9,00 & 2,00 & 2,90 & 2,42 & \\
\hline \multirow{2}{*}{ TPD3 } & G1 & 4,00 & 10,00 & 10,00 & 9,02 & 1,71 & \multirow{2}{*}{$0,02^{*}$} & 1,00 & 10,00 & 9,00 & 8,02 & 2,42 & \multirow{2}{*}{0,32} \\
\hline & G2 & 1,00 & 10,00 & 9,00 & 7,97 & 2,73 & & 1,00 & 10,00 & 8,00 & 7,45 & 2,66 & \\
\hline \multirow{2}{*}{ TPD4 } & G1 & 3,00 & 10,00 & 10,00 & 8,73 & 1,96 & \multirow{2}{*}{$0,01^{*}$} & 0,00 & 10,00 & 9,00 & 7,90 & 2,65 & \multirow{2}{*}{0,25} \\
\hline & G2 & 2,00 & 10,00 & 9,00 & 7,72 & 2,46 & & 0,00 & 10,00 & 8,00 & 7,24 & 2,84 & \\
\hline
\end{tabular}

Teste de Mann -Whitney

Legenda:

G1: Grupo sem alteração fonoaudiológica

G2: Grupo com alteração fonoaudiológica

TPF3: Teste de padrão tonal de frequência com três sons

TPF4: Teste de padrão tonal de frequência com quatro sons

DP: Desvio padrão 
$\mathrm{Na}$ análise da variável sexo e TPD e TPF, verificou-se que em ambos os testes a média de acertos foi maior no sexo masculino (Figuras 2 e 3). Cabe considerar, o número distinto de sujeitos de cada sexo.

Com a associação entre questão 1 do formulário e TPF3 (nomeação e símbolos), no ambiente silencioso e ruidoso, foi possível verificar que a média nesse teste, entre as alternativas da questão 1 (baixa iluminação, excesso de poeira, falta de ventilação, mobiliário inadequado, ruído e temperatura) foi maior no grupo sem alteração fonoaudiológica (G1) (Figuras 4 e 5). Esse mesmo padrão pode ser observado no TPD. Quanto à percepção do ruído, o desempenho de G1 e G2 foi semelhante, demonstrando a interferência do ruído nos dois grupos estudados.

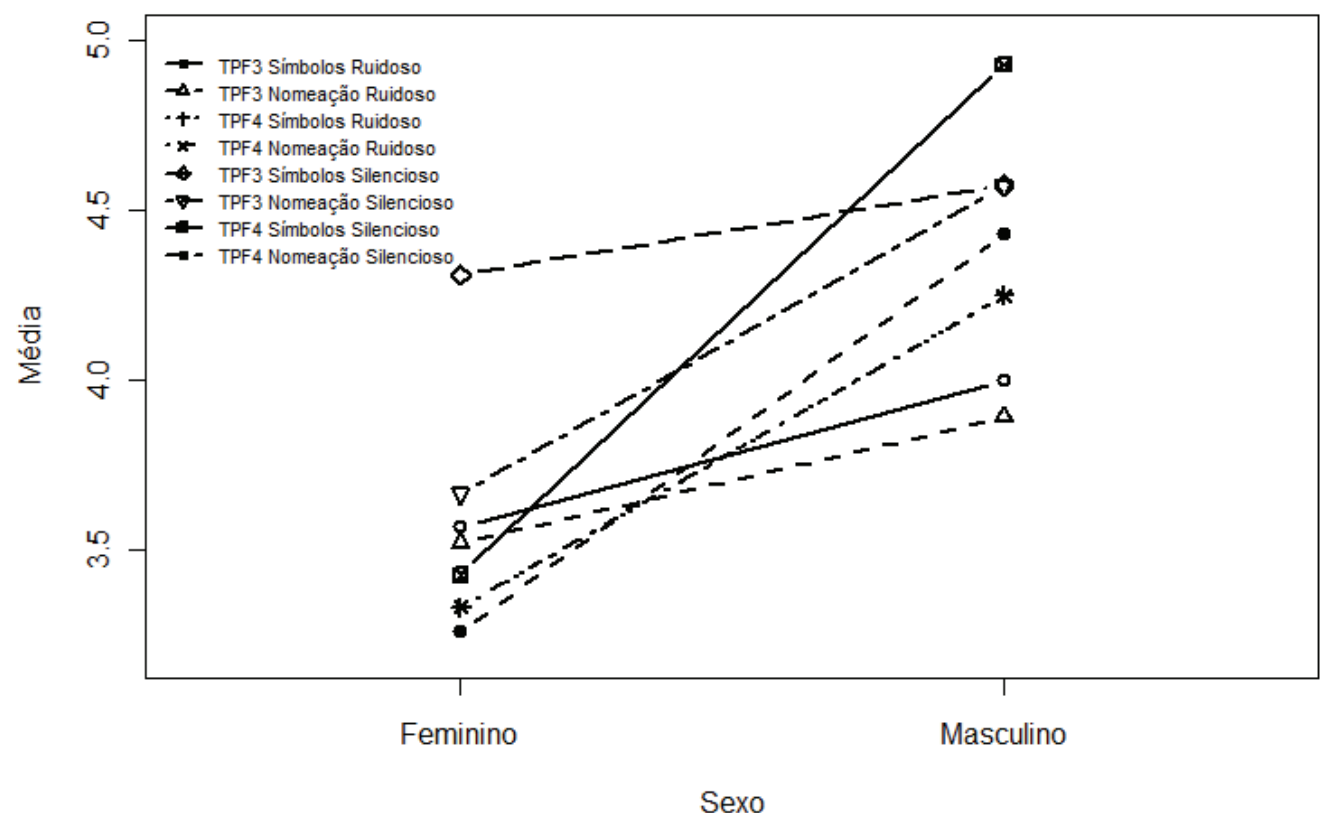

Figura 2 - Gráfico representativo da associação entre a variável sexo e desempenho no teste de padrão tonal de frequência - símbolos e nomeação em ambiente silencioso e ruidoso

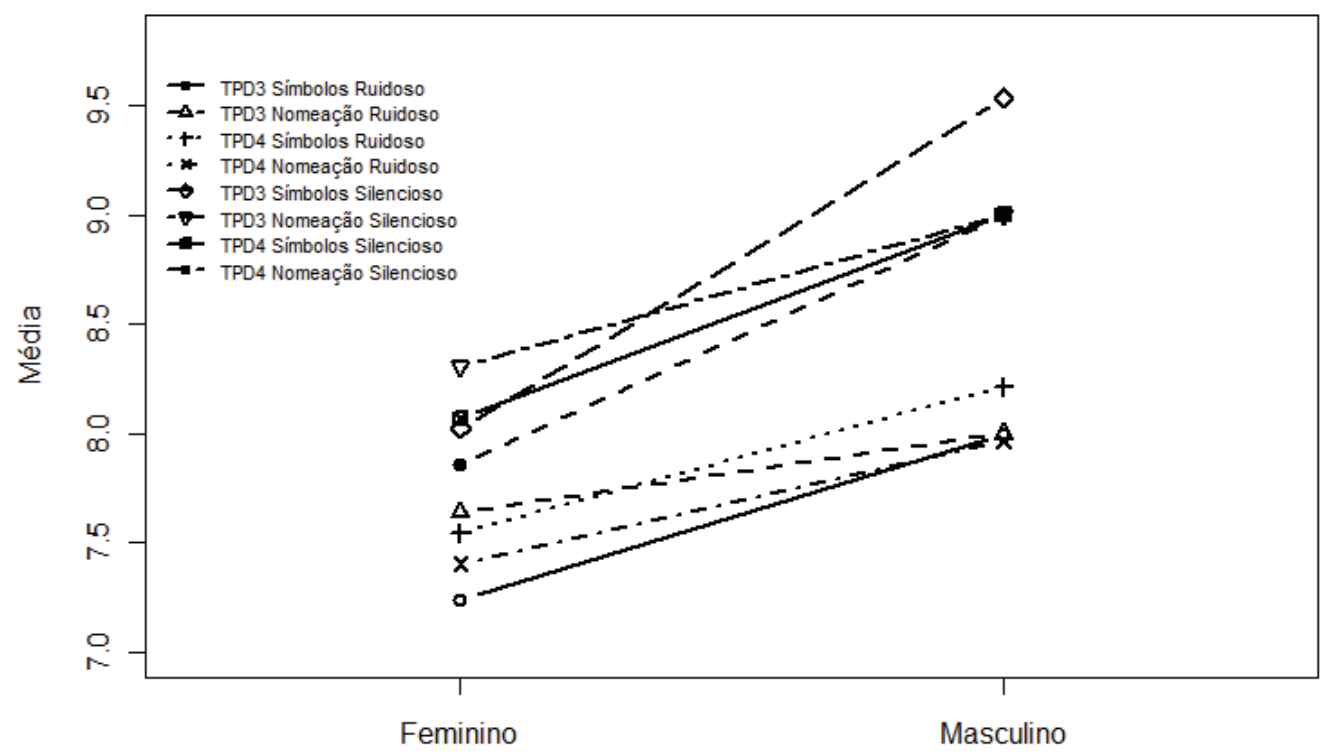

Sexo

Figura 3 - Gráfico representativo da associação entre a variável sexo e o desempenho no teste de padrão tonal de duração - símbolos e nomeação em ambiente silencioso e ruidoso 


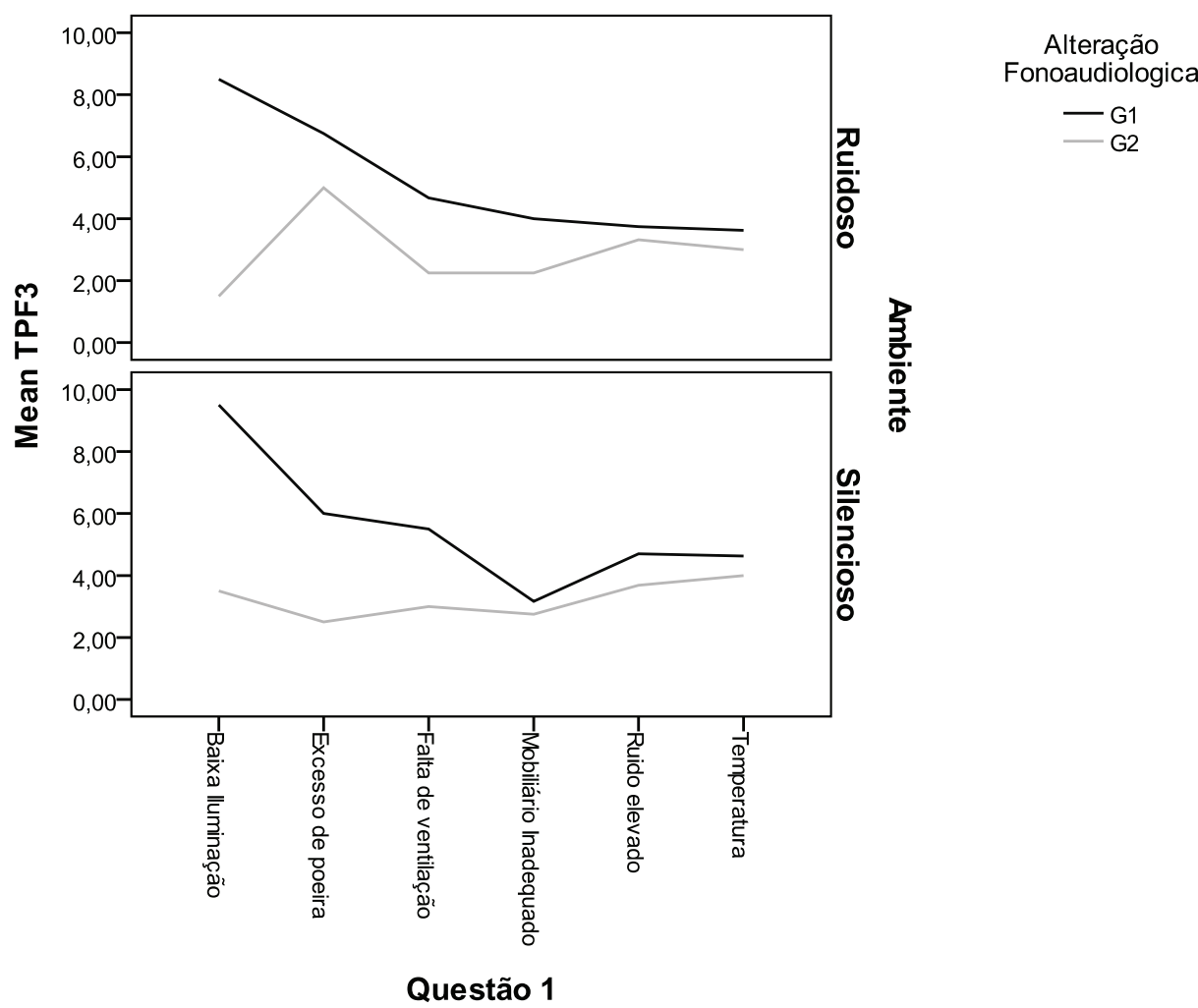

Figura 4 - Gráfico representativo da associação entre o TPF3, ambiente de testagem (silencioso/ ruidoso), grupos de estudo (G1 e G2) e alternativas da questão 1

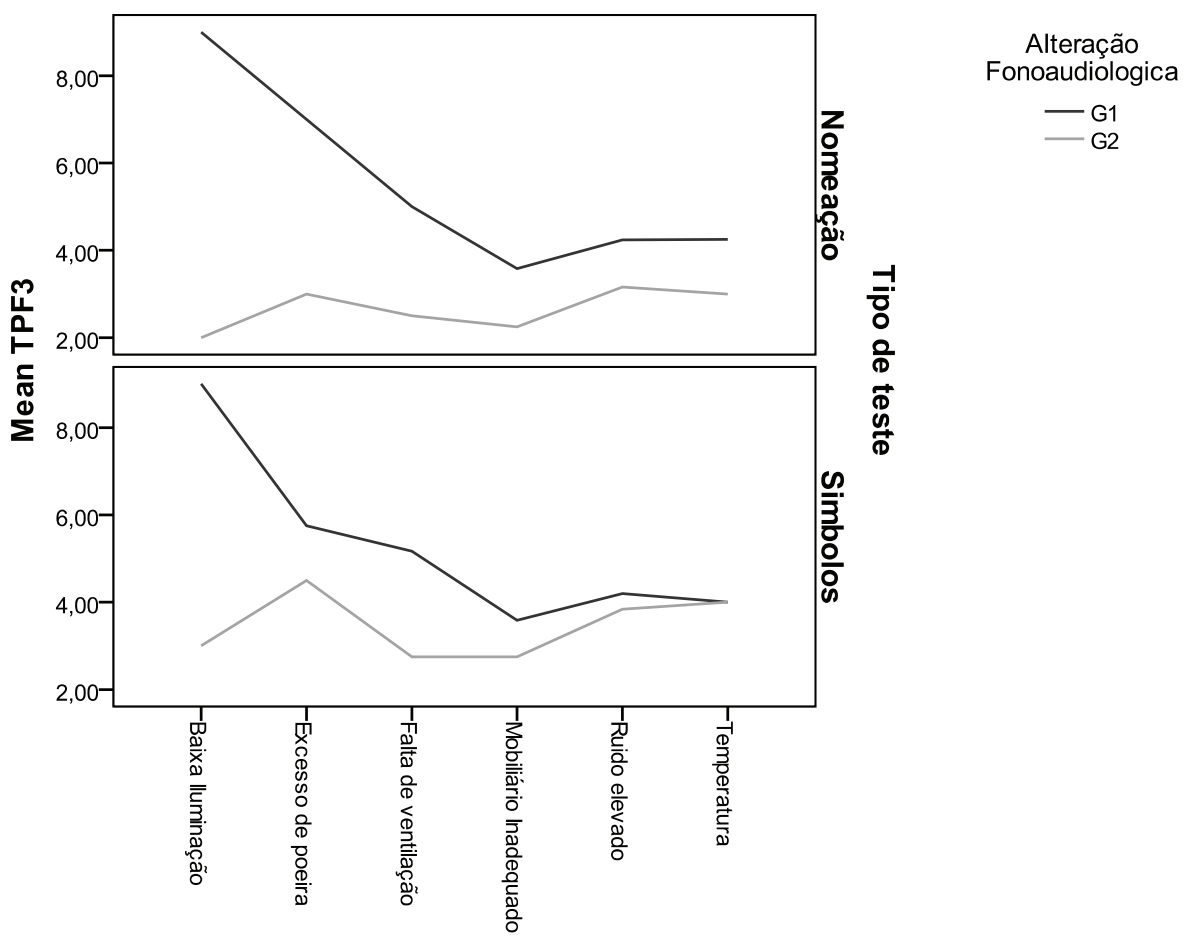

Questão 1

Figura 5 - Gráfico representativo da associação entre o TPF3 (símbolos e nomeação), grupos de estudo (G1 e G2) e alternativas da questão 1 


\section{DISCUSSÃO}

Cada indivíduo se refere a um mesmo ruído competidor de diferentes formas ${ }^{9}$. Os estudantes classificaram o ruído interno da escola como elevado, gerando grande interferência nas suas atividades.

Em relação à percepção dos estudantes, os achados do presente estudo corroboraram as pesquisas que citaram 0 barulho de pessoas conversando como a causa de maior interferência em sala de aula 18,19. A conversa de estudantes durante a realização de atividades é a fonte dominante de ruído nas salas de aula ativas ${ }^{20}$. Além disso, um ambiente escolar ruidoso pode prejudicar a compreensão da mensagem ${ }^{21}$ e a capacidade de aprendizagem dos estudantes $9,13,22,23$.

Quanto à avaliação do ruído escolar, os achados desse estudo corroboraram pesquisas que evidenciaram níveis de ruído acima do estabelecido pelas normas nacionais ${ }^{11,13,19,24}$, contrapondo o recomendado pela norma brasileira NBR 10.152 que estabelece que o ruído em sala de aula deve variar entre $40 \mathrm{~dB}(\mathrm{~A})$ e $50 \mathrm{~dB}(\mathrm{~A}){ }^{25}$. Esse é um dado preocupante, visto que o ruído pode causar vários efeitos adversos à saúde humana ${ }^{1,2}$, comprometendo a qualidade de vida de estudantes, professores e funcionários.

A utilização de formulários de percepção do ruído ambiental no ambiente escolar mostrou-se um instrumento eficiente na detecção das principais fontes de ruído, corroborando com a avaliação objetiva dos níves sonoros, revelando que a percepção do ruído pode ser confirmada por meio de medições físicas ${ }^{26}$.

Considerando que a comunicação ocorre em ambiente social, com outros estímulos competitivos ${ }^{27}$, os testes de padrão tonal de frequência e padrão tonal de duração foram realizados em escuta diótica, a fim de pesquisar a extensão do problema de comunicação ocasionado pela interferência do ruído. Tais testes podem ser realizados em campo livre e a importância e efetividade de sua realização foram descritas na literatura nacional ${ }^{17}$

Nesse estudo, os testes de ordenação temporal foram realizados sem avaliação da audição periférica dos estudantes, realizou-se somente a pesquisa do reflexo cócleopalpebral (RCP) que esteve presente em $100 \%$ da amostra. O que pode garantir a exclusão de problemas condutivos, ou de perdas auditivas severas e profundas bilaterais ${ }^{14}$.

Em relação ao ambiente de testagem, quanto mais positiva a relação sinal/ruído se apresentou, melhor situação de escuta foi oferecida. Quanto mais próxima ao zero ou negativa a relação sinal/ruído, pior a situação para a compreensão da mensagem ${ }^{28}$.

Nos testes de padrão tonal de frequência (TPF) e padrão tonal de duração (TPD), os achados desse estudo corroboraram com as pesquisas que verificaram o ruído afetando a realização de testes ${ }^{27}$ e tarefas cotidianas ${ }^{29}$,aumentando o número de erros. Contudo vale lembrar que parte dos autores ${ }^{27}$ não utilizou o mesmo teste que o presente estudo. Apenas um estudo ${ }^{17}$ utilizou os mesmos testes e metodologia da presente pesquisa em escolares, porém sem verificar a influência do ruído na aplicação do TPF e TPD. Não foram encontrados na literatura pesquisada estudos semelhantes que utilizaram testes de ordenação temporal em diferentes ambientes de testagem. A média de acertos foi maior no TPD do que no TPF, corroborando com a literatura nacional ${ }^{17}$, indicando que o padrão de duração foi mais fácil que o de frequência. No entanto, na literatura internacional foi verificado estudo utilizando outra proposta de testes no qual o padrão de duração teve mais erros quando comparado ao padrão de frequência ${ }^{30}$.

Quanto à análise do TPF e TPD e os grupos G1 e G2, observou-se que as médias nesses testes, tanto na modalidade de resposta por símbolos quanto por nomeação foram maiores no grupo sem alteração fonoaudiológica (G1), que apresentou adequação em todas as provas realizadas, a saber: fala, motricidade orofacial, linguagem e avaliação simplificada do processamento auditivo. Verificouse esse mesmo padrão no ambiente silencioso e no ruidoso. Este dado remete a literatura que refere que indivíduos com alterações da comunicação humana são de risco ou apresentam maiores evidências de alterações de processamento auditivo ${ }^{12,17,31}$.

Resultado semelhante ao do presente estudo quanto ao desempenho de grupos foi encontrado em estudo nacional, sem o envolvimento do ruído ambiental, no qual os indivíduos do grupo com desempenho alterado na avaliação fonoaudiológica apresentaram prejuízo em ordenação temporal de sons com diferentes frequências e durações ${ }^{31}$. A ordenação temporal de padrões sonoros foi avaliada em crianças com e sem déficit de processamento fonológico, utilizando como critério de classificação dos grupos a prova de consciência fonológica. Os indivíduos foram submetidos à realização do teste de padrão de duração e frequência em cabina acústica ${ }^{31}$.

É preciso considerar que o presente estudo envolveu experimento realizado in loco, ou seja, no ambiente escolar e que deste modo a preocupação em alterar minimamente a rotina e o contexto escolar foram intervenientes importantes para a diminuição do protocolo de inclusão dos sujeitos. 
Assim, optou-se por realizar a avaliação simplificada de processamento auditivo. Tal procedimento garantiu apenas a adequação das habilidades de localização sonora e ordenação temporal simples para sons verbais e não verbais. Obviamente, não há garantia de que os sujeitos incluídos no grupo 1 estivessem livres de alterações de outras habilidades do processamento auditivo. Contudo, é certo que a ordenação simples tanto para sons verbais quanto não verbais mostrou-se adequada nestes sujeitos, garantindo a possibilidade da discussão das alterações de ordenação temporal complexa.

Os resultados demonstraram que mesmo os sujeitos sem alterações fonoaudiológicas tiveram pior desempenho no ruído. Essa piora no desempenho evidenciou a interferência do ruído no processamento da informação via audição ${ }^{27,32}$.

$\mathrm{Na}$ análise da variável sexo, foi descrita na literatura ${ }^{33}$ diferença com significância estatística entre os sexos, sendo que o sexo masculino apresentou melhor desempenho em testes de resolução temporal. Na literatura internacional ${ }^{34}$ também foram encontrados resultados sugestivos de que crianças do sexo masculino apresentavam limiares de resolução temporal mais baixos (melhores) que os de crianças do sexo feminino. Outros autores ${ }^{35-36}$ verificaram ausência de diferenças com significância estatística nas habilidades de processamento temporal entre os sexos. No presente estudo foi observado melhor desempenho para o sexo masculino, o que corrobora parte da literatura pesquisada ${ }^{37}$ que também utilizou os testes de padrão tonal de frequência e duração em uma amostra de 80 indivíduos adultos jovens, sendo quarenta do sexo masculino e quarenta do sexo feminino.

Observou-se que quanto maior a percepção de interferência do ruído pelo indivíduo, pior foi o desempenho nos testes de padrão tonal de frequência e padrão tonal de duração. Não foram encontrados estudos que pesquisaram o mapeamento de locais ruidosos no ambiente escolar e sua relação com o desempenho em testes do processamento auditivo.

Assim, foi possível verificar que os sujeitos que perceberam menos o ruído da escola tenderam a apresentar melhor desempenho nos testes de padrão tonal de frequência e duração, independentemente de apresentarem alterações fonoaudiológicas ou não. Tal achado pode ser explicado pelas habilidades de fechamento e figura-fundo. Os sujeitos que tiveram melhor desempenho no teste devem ter melhor capacidade de isolar o ruído de fundo e dar atenção a mensagem principal.
Conhecendo-se os efeitos prejudiciais do excesso de ruído para a saúde e desempenho escolar dos estudantes, percebeu-se a importância de níveis sonoros adequados no ambiente escolar. Uma vez que, inúmeras fontes geradoras de ruído estão presentes nas escolas, sejam elas internas ou externas ${ }^{9}$.

Outro dado relevante foi o fato de que a modalidade de respostas dos testes, símbolos ou nomeação, não influenciou no desempenho, evidenciando que em uma avaliação, que utilize o TPF e TPD em escolas, pode ser utilizada qualquer uma das modalidades reposta.

Nesse estudo, a avaliação do processamento da informação via audição em um ambiente "natural" de aprendizagem, a própria escola, é grande avanço para a investigação das desordens do processamento auditivo, visto que avaliou o estudante em situação real de exigência, com as dificuldades inerentes ao seu cotidiano. Apesar das contribuições do estudo, foram observadas algumas limitações durante a realização do mesmo. Uma delas foi a utilização de amostra de conveniência em lugar de amostra de base populacional. $E$ também um número desigual entre estudantes do sexo masculino e feminino, que dificultou a realização de análises entre a variável sexo e o desempenho nos testes realizados. A amostra pequena dificultou a realização de análises que envolvessem a análise do desempenho (normal/alterado) nos testes de padrão tonal de frequência e padrão tonal de duração correlacionado com outras variáveis desse estudo.

Vale citar a ausência de uma avaliação da audição periférica, importante para detectar alguma alteração auditiva leve, visto que foi realizada somente a pesquisa do reflexo cócleopalpebral (RCP) que pode descartar problemas condutivos, ou perdas auditivas severas e profundas ${ }^{14}$. Desse modo, acredita-se que é importante realizar uma avaliação da audição periférica formal, todavia nem sempre isso é possível no ambiente escolar, sendo a aplicação de testes em campo livre uma alternativa para a avaliação de escolares no seu ambiente de aprendizagem.

Um achado preocupante desse estudo foi o nível elevado do ruído no ambiente escolar, prejudicando os estudantes em suas atividades acadêmicas. O problema vai além da percepção do incômodo causado pelo ruído, exige reflexões quanto à distribuição física do ambiente escolar, acústica das salas de aula e conscientização dos estudantes e funcionários em relação a geração do ruído interno da escola. 


\section{CONCLUSÕES}

Nos Testes de padrão tonal de frequência e padrão tonal de duração, observou-se que a média de acertos no ambiente silencioso foi maior que no ambiente ruidoso. O grupo sem alteração fonoaudiológica (G1) apresentou melhor desempenho que o grupo com alteração fonoaudiológica (G2). No ruído, houve piora no desempenho dos dois grupos estudados, ou seja, mesmo sujeitos sem alteração fonoaudiológica apresentaram pior desempenho no ruído, demonstrando a grande interferência de níveis sonoros elevados no processamento da informação via audição.

\begin{abstract}
Purpose: to characterize student performance in Frequency Pattern Test (FPT) and Duration Pattern Test (DPT) in a silent ambient and under noise. Method: experimental cross-sectional study, measured in a convenience sample. Survey made up by 70 students who answered a form on their noise perception. School's noise level was evaluated using physical measurements. Students were submitted to previous evaluations (speech, language, and auditory processing) and then splinted into two groups (G1 - with no Speech-language pathology alteration; G2 - with Speech-language pathology alteration). Students were also submitted to Frequency and Duration Pattern Tests (in silence under noise). Results: school's sound pressure level ranged from $57.2 \mathrm{dBA}$ in school's computer lab to 83.6 dBA in school's gym. According to students, what interferes most in their activities is the buzz from conversations during class. Regarding FPT and DPT, it was possible to note that score average in a quiet environment was higher than under noise. G1 had better performance in the FPT and DPT than G2. Noise worsened in both groups (G1 and G2) as for the performance in tests. Conclusion: school's sound pressure levels are high and out of standard recommendations under the Brazilian national norms. Noise worsened both group performances in FPT and DPT.
\end{abstract}

KEYWORDS: Noise; Hearing Tests; Learning; Noise Effects; Auditory Perception

\section{REFERÊNCIAS}

1. World Health Organization (WHO). Noise, environmental health criteria. Geneva, Swz, 1980. Disponível em http://www.inchem.org/documents/ ehc/ehc/ehc012.htm Acesso em: 05 Abril 2009.

2. Lacerda ABM, Magni C, Morata TC, Marques JM, Zannin PHT. Ambiente urbano e percepção da poluição sonora. Ambiente. Soc. 2005; 8 (2): 85-98. 3. Clark C, Stansfeld SA. The effect of Transportation Noise on Health and Cognitive development: A Review of Recent Evidence. International Journal of Comparative Psychology. 2007; 20(2): 145-58.

4. Moller LP, Iniguez R, Cevo J, Ayala F. Medición de los niveles de ruido ambiental en la ciudad de Santiago de Chile. Rev. Otorrinolaringol. Cir. Cabeza Cuello. 2007; 67: 122-8.

5. Santos JD, Ferreira MIDC. Variação dos limiares audiométricos em trabalhadores submetidos ao ruído ocupacional. Arq. Int. Otorrinolaringol. 2008; 12 (2): 201-9.

6. Gonçalves CGO, Mota PHM, Marques JM. Ruído e idade: análise da influência na audição em indivíduos com 50-70 anos. Pró-fono R. Atual. Cient. 2009; 21(4): 57-62.
7. Lopes AC, Nelli MP, Lauris JRP, Amorim RB, Melo ADP. Condições de saúde auditiva no trabalho: Investigação dos Efeitos auditivos em trabalhadores expostos ao ruído ocupacional. Arq. Int. Otorrinolaringol. 2009; 13(1): 49-54.

8. Zocoli AMF, Morata TC, Marques JM. Adaptação para 0 português brasileiro do questionário: Youth Attitude to noise scale (YANS). Braz J Otorhinolaryngol. 2009; 75 (4): 485-92.

9. Dreossi RCF, Momensohn-Santos T. O ruído e sua interferência sobre estudantes em uma sala de aula: revisão de literatura. Pró-fono revista brasileira de Atualização Científica. 2005; 17 (2): 251-8.

10. Estrada-Rodriguez C, Ramírez IM. Impacto del ruido ambiental en estudiantes de educación primaria de la ciudad de México. Revista Latinoamericana de Medicina Conductal. 2010; 1(1): 57-68.

11. Ribeiro MER, Oliveira RLS, Santos TMM, Scharlach RC. A percepção dos professores de uma escola particular de Viçosa sobre o ruído nas salas de aula. Rev. Equilíbrio Corporal e saúde. 2010; 2 (1): 27-45.

12. American Speech-Language-Hearing Association. Central Auditory Processing: Current Status of Research and Implications for Clinical Practice 
[Technical Report]. 1996. Disponível em: http:// www.asha.org/policy

13. Eniz AO, Garavelli SL. A contaminação acústica de ambientes escolares devido aos ruídos urbanos no Distrito Federal. Holos Environment. 2006; 6: 137-50.

14. Momensohn-Santos TM. Perda auditiva funcional. In: Filho OL. Tratado de Fonoaudiologia. São Paulo: Tecmed; 1997. p. 153-71.

15. Associação Brasileira de Normas Técnicas (ABNT). NBR 10151. Acústica: Avaliação do ruído em áreas habitadas, visando o conforto da comunidade - procedimento. Rio de Janeiro; 2000.

16. Schweitzer HCS. Considerações Binaurais e direcionais para a reabilitação auditiva. In: Iorio MCM, Almeida $\mathrm{K}$ de. Próteses auditivas: Fundamentos teóricos e aplicações clínicas. São Paulo: Lovise; 2003. p. 95-117.

17. Gimenes VD. Identificação de padrões acústicos de duração e de frequência e habilidade grafofônica [tese]. São Paulo: Universidade Federal de São Paulo; 2000.

18. Lundquist $\mathrm{P}$, Holmberg $\mathrm{K}$, Landström $\mathrm{U}$. Annoyance and effects on work from environmental noise at school. Noise Health. 2000; 2(8): 39-46.

19. Jaroszewski GC, Zeigelboiim BS, Lacerda A. Ruído escolar e sua implicação na atividade de ditado. Rev. CEFAC. 2007; 9 (1): 122-32.

20. Sato $H$, Bradley JS. Evaluation of acoustical conditions for speech communication in working elementary school classrooms. J. Acoust Soc. Am. 2008; 123(4): 2064-77.

21. Henriques MO, Miranda EC de, Costa MJ. Limiares de reconhecimento de sentenças no ruído, em campo livre: valores de referência para adultos normo-ouvintes. Rev Bras de Otorrinolaringol. 2008; 74 (2): 188-92.

22. Haines MM, Stansfeld SA, Head J, Job RFS. Multilevel modelling of aircraft noise on performance tests in schools around Heathrow Airport London. J Epidemiol Community Health 2002. 56: 139-44.

23. Clark C, Martin R, Kempen EV, Alfred T, Head J, Davies HW et al. Exposure-Effect relations between

http://dx.doi.org/10.1590/S1516-18462011005000093

RECEBIDO EM: 04/11/2010

ACEITO EM: 06/04/2011

Endereço para correspondência:

Stela Maris Aguiar Lemos

Av. Prof. Alfredo Balena, 190, sala 069 -

Faculdade de Medicina da UFMG

Belo Horizonte, MG

CEP: 30130-100

E-mail: smarislemos@medicina.ufmg.br aircraft and road traffic noise exposure at school and reading comprehension. Am J Epidemiol. 2006; 163 (1): 27-37.

24. Libardi A, Gonçalves CG de O, Vieira TPG, Silverio KCA, Rossi D, Penteado RZ. O ruído em sala de aula e a percepção dos professores de uma escola de ensino fundamental de Piracicaba. Distúrbios da Comunicação. 2006; 18 (2): 167-78.

25. Associação Brasileira de Normas Técnicas (ABNT). NBR 10152: Níveis de ruído para conforto acústico. Rio de Janeiro; 1987.

26. Paz EC da, Ferreira AMC, Zannin PHT. Estudo Comparativo da percepção do ruído urbano. Rev saúde pública. 2005; 39 (3): 467-72.

27. Caporali SA, Silva JA da. Reconhecimento de fala no ruído em jovens e idosos com perda auditiva. Rev Bras Otorrinolaringol. 2004; 70(4): 525-32.

28. Soncini F, Costa MJ. Efeito da prática musical no reconhecimento da fala no silêncio e no ruído. Pró-fono revista de atualização científica. 2006; 18 (2): 161-70.

29. Miranda JRC. Ruído: efectos sobre la salud y criterio de su evaluación al interior de recintos. Revista ciencia y trabajo. 2006; 8 (20): 42-6.

30. Bellis TJ. Assessment and management of Central Auditory Disorders in the Educational setting: from science to practice. Canada: Thomson Delmar Learning; 2003.

31. Frota SMMC, Pereira LD. Processos temporais em crianças com déficit de Consciência Fonológica. Revista Iberoamericana de Educácion. 2004; 2: 1-11.

32. Calais LL, Russo ICP, Borges ACLC. Desempenho de idosos em um teste de fala na presença de ruído. Pró-fono Revista de Atualização Científica. 2008; 20 (3): 147-52.

33. Zaidan E, Garcia AP, Tedesco MLF, Baran JA. Desempenho de adultos jovens normais em dois testes de resolução temporal. Pró-fono Revista de Atualização Científica. 2008; 20 (1): 19-24.

34. Davis SM, Mccroskey RL. Auditory fusion in children. Child Dev. 1980; 51: 75-80.

35. Ahmmed A et al. Auditory temporal resolution in children with specific language impairment. Journal of Medical Speech-Language Pathology. 2006; 14(2): 79-96.

36. Muniz LF, Roazzi A, Schochat E, Teixeira CF, Lucena JA. Avaliação das habilidades de resolução temporal, com uso de tom puro, em crianças com e sem desvio fonológico. Rev Cefac. 2007; 9(4): 550-2.

37. Corazza MC. Avaliação do processamento auditivo central em adultos: teste de padrões tonais auditivos de frequência e testes de padrões tonais auditivos de duração [tese]. São Paulo: Universidade Federal de São Paulo; 1998. 